\title{
A Retrospective Cohort Study in a University Hospital Evaluating the Effect of Maternal Glucose- Containing Intravenous Fluid in Neonatal Glycemia
}

\author{
Gelinemae G. Malaza, M.D.', \\ Elaine C. Cunanan, M.D. ${ }^{1,2}$
}

Abstract Maternal hyperglycemia during the peripartum period is highly correlated with fetal hyperinsulinemia and consequent neonatal hypoglycemia. Liberal use of intravenous glucose therapy prior to delivery can potentially cause maternal hyperglycemia, therefore, it is prudent to implement all preventive measures.

This study aims to determine the occurrence of neonatal hypoglycemia with intravenous glucose therapy prior to delivery in maternal diabetes mellitus.

This was a retrospective cohort study of neonates born from diabetic mothers at the University of Santo Tomas Hospital Clinical and Private Divisions from January 1, 2013 to December 15, 2017. Clinical information gathered was divided into maternal and neonatal characteristics. Maternal intravenous fluid use, rate, and duration were noted; maternal and neonatal blood glucose results were obtained.

There were 109 infants of diabetic mothers, of which 105 were delivered as singleton and 4 from

Gelinemae Malaza

ggm2185@yahoo.com

1 Section of Endocrinology, Department of Internal Medicine, University of Santo Tomas Hospital, Manila, Philippines

2 Faculty of Medicine and Surgery, University of Santo Tomas, Manila, Philippines twin pregnancies. Neonatal hypoglycemia was present in $14.68 \%$. Comparing the risk factors, there was a higher amount of glucose infused to the mothers whose offspring developed hypoglycemia compared to those without hypoglycemia. Statistically, this did not demonstrate a significant difference. The rate of glucose infusion and frequency of maternal insulin use were similar between the groups. Linear correlation was not evident when the total glucose infused and the rate of intravenous glucose infusion was compared to the neonatal glucose in the first hour of life.

Based on this study, routine administration of glucose-containing intravenous fluid did not influence the incidence of neonatal hypoglycemia. It is recommended that further prospective studies be conducted.

Keywords gestational diabetes mellitus, neonatal hypoglycemia, intravenous fluid

\section{INTRODUCTION}

Maternal diabetes mellitus is associated with increased risk of fetal and neonatal complications, with neonatal hypoglycemia occurring in $8 \%$ to $30 \%$ [1]. Neonatal hypoglycemia screening in all newborns from diabetic mothers is mandatory because of its 
immediate and long-term consequences, having glucose as the primary substrate for the brain [2].

Intravenous fluid (IVF) use prior to delivery provides hydration and caloric supplementation to parturient women and may reduce the duration of labor and cesarian section [3]. It serves to supplement the decreased hepatic supply of glucose during the active phase of labor [4] and provide adequate maternal hydration in the setting of higher metabolic demand [3].

The maternal hyperglycemia-fetal hyperinsulinemia hypothesis proposed by Pedersen [5] in 1952 has been the cornerstone in the pathophysiology of neonatal hypoglycemia. Maternal hyperglycemia during the peripartum period is highly correlated with fetal hyperinsulinemia and consequent neonatal hypoglycemia $[4,5,6]$. With the interruption of maternal-fetal circulation upon delivery, there is likewise cessation of maternal transfer of glucose. Elevated circulating insulin coupled with a decrease in substrate is one mechanism of hypoglycemia in infants of diabetic mothers [1].

Earlier studies have shown that neonatal hypoglycemia is correlated with the presence of glucose infusion before a cesarian section [7] and maternal intravenous glucose therapy duration, total amount of glucose received, and rate of glucose per hour [8]. Some institutions use intravenous glucose supplementation only when the duration of labor exceeds 8 hours [5], whereas others use glucose infusion with simultaneous insulin infusion or rotating fluid with continuous glucose and non-glucose drip [9]. These aim to maintain maternal capillary plasma glucose within the normal range between 4-7 $\mathrm{mmol} / \mathrm{L}(72-126 \mathrm{mg} / \mathrm{dL}$ ) [10] since blood glucose elevations within 4 to 6 hours prior to delivery increase the rate of neonatal hypoglycemia [11].

There is no local guideline available on the use of glucose-containing intravenous fluids in the peripartum period. Liberal use of intravenous glucose therapy prior to delivery can cause maternal hyperglycemia. Therefore, it is prudent to implement all measures to prevent such from happening.

This study aims to determine the occurrence of neonatal hypoglycemia with intravenous glucose therapy to parturient women prior to giving birth. Specifically, we sought to determine the significant difference on the amount of glucose infused to the parturient women between neonates who developed hypoglycemia versus those without hypoglycemia, identify the correlation between the glucose infused to the mother prior to delivery and neonatal blood glucose in the first hour of life, and to identify risk factors in the development of neonatal hypoglycemia.

\section{METHODOLOGY}

\section{Study Design}

This was a retrospective cohort study of neonates born from diabetic mothers.

\section{Database Population}

Women with diabetes mellitus during pregnancy and their neonates delivered in the University of Santo Tomas Hospital (USTH) Clinical and Private Divisions from January 1, 2013 to December 15, 2017 were included in the study. This time frame was selected based on the available registry of gestational and pre-gestational diabetes mellitus who delivered at the USTH. The total enumeration method was employed in gathering data. Medical records of patients with maternal age 18 years old and above and with available information on maternal and neonatal characteristics and laboratory data were retrieved, whereas patients with maternal type 1 diabetes mellitus and incomplete records were excluded from the study. Clinical information was divided into maternal and neonatal characteristics. Maternal characteristics included maternal age, gestational week when diabetes was diagnosed, medications for glycemic control, number of hours on nothing per orem (NPO) prior to delivery, and IVF used, its rate, duration and total amount of glucose received prior to delivery. Neonatal characteristics included a method of delivery, gender, maturity testing and birth weight. Maternal laboratory data included capillary blood glucose levels while on NPO within 6 hours prior to delivery. Neonatal laboratory data included heelstick glucose determination results within the first 24 hours of life. Glucose level less than the $10^{\text {th }}$ percentile $(2.2 \mathrm{mmol} / \mathrm{L}$ or $39.6 \mathrm{mg} / \mathrm{dL})$ on the first day of life [5] was grouped under neonatal hypoglycemia whereas those who did not fulfill this criterion were grouped under without neonatal hypoglycemia. 


\section{Data Management and Statistical Analysis}

Data were encoded into the computer using the Microsoft $[$ Excel software. Frequencies were reported as percentages. Prospective risk factors were grouped into maternal glycemic influence and other risk factors. Categorical variables such as maternal intravenous glucose and insulin use, method of delivery, and offspring gender and symmetry were reported as percentage whereas numerical variables such as amount of glucose infused per hour, total glucose infused, maternal blood glucose, maternal age, gestational week, maturity testing, and neonatal birth weight were reported as mean \pm standard deviation. Numerical variables were compared using student t-test while categorical variables were compared using the chi-square test. Pearson correlation was done to determine the association between neonatal glucose on the first hour of life versus grams of glucose infused per hour and total glucose infused on the mother. P-value of less than 0.05 was considered significant.

\section{Results}

A total of 123 infants of diabetic mothers were reviewed and 109 were included in the study. Of these, 105 were delivered as singleton presentations and 4 were from twin pregnancies. Neonatal hypoglycemia was present in $14.68 \%(\mathrm{~N}=16)$ of the infants of diabetic mothers. The use of intravenous glucose therapy was employed in $95.41 \%(\mathrm{~N}=104)$. Insulin for maternal glycemic control was used in $28.9 \%$ (31 of 107 mothers) and was not statistically significant between those whose offsprings developed neonatal hypoglycemia and those without hypoglycemia.

Comparing the risk factors as to maternal glycemic influence (Table 1), there was a higher amount of glucose infused to the mothers whose offspring developed hypoglycemia compared to those without hypoglycemia. The latest maternal capillary blood glucose was also higher in the group with neonatal hypoglycemia. Statistically, these did not demonstrate a significant difference. The rate of glucose infusion and frequency of maternal insulin use were similar between the two groups.

As to the other risks that may influence the development of neonatal hypoglycemia (Table 2), the maternal age, gestational week at diagnosis of diabetes mellitus, method of delivery, offspring gender, maturity testing, and neonatal birthweight and symmetry did not show significant differences as well. Notably, among neonates with hypoglycemia, there were more cesarian deliveries and more male offsprings delivered.

Linear correlation was not evident when the total glucose infused was compared to the neonatal glucose in the first hour of life $(r=0.0643$, $p$-value $=$ $0.5065)$. Similarly, the rate of intravenous glucose infusion did not show linear correlation with neonatal glucose in the first hour of life.

Comparing the maternal blood glucose within 6 hours prior to delivery in neonates who developed hypoglycemia versus those without hypoglycemia, there is a higher trend of maternal capillary blood glucose in those whose neonates developed hypoglycemia and this showed statistical significance with a p-value of 0.0072 .

\section{Discussion}

Long-term neurodevelopmental dysfunction has been associated with hypoglycemic neonates [14], hence it is imperative to implement all measures to prevent its occurrence. A systematic review of 16 articles categorized the risk factors of neonatal hypoglycemia into infant-related and mother-related [1]. Infantrelated significant risk factors were small and large

Table 1. Comparison of maternal glycemic influence in neonates who developed hypoglycemia on the first hour of life versus neonates without hypoglycemia

\begin{tabular}{llll}
\hline Risk Factor & $\begin{array}{l}\text { With Neonatal Hypoglycemia, } \\
\mathrm{N}=16 \text { (Mean+SD or Percentage) }\end{array}$ & $\begin{array}{l}\text { Without Neonatal Hypoglycemia, } \\
\mathrm{N}=93 \text { (Mean+SD or Percentage) }\end{array}$ & p-value \\
\cline { 2 - 4 } Intravenous glucose use & $87.5 \%, \mathrm{~N}=14$ & $96.77 \%, \mathrm{~N}=90$ & 0.1033 \\
Maternal insulin use & $31.25 \%, \mathrm{~N}=5$ & $30.11 \%, \mathrm{~N}=28$ & 0.9273 \\
$\begin{array}{l}\text { Amount of glucose infused per } \\
\text { hour (g/hr) }\end{array}$ & 6 & $5.61 \pm 1.03$ & 0.3686 \\
Total glucose infused from IVF (g) & $61.4 \pm 144.31$ & $34.66 \pm 31.55$ & 0.2560 \\
Latest maternal CBG (mg/dL) & $103.93 \pm 26.96$ & $93.87 \pm 22.67$ & 0.1026 \\
\hline
\end{tabular}

p-value $<0.05$, significant 
Table 2. Comparison of other risk factors in neonates who developed hypoglycemia on the first hour of life versus neonates who did not develop hypoglycemia

\begin{tabular}{|c|c|c|c|}
\hline Risk Factor & $\begin{array}{l}\text { With Neonatal Hypoglycemia, } \\
\text { N=16 (Mean+SD or Percentage) }\end{array}$ & $\begin{array}{l}\text { Without Neonatal Hypoglycemia, } \\
\text { N=93 (Mean+SD or Percentage) }\end{array}$ & p-value \\
\hline Maternal age (years) & $30.06 \pm 5.77$ & $31.36 \pm 5.49$ & 0.2053 \\
\hline $\begin{array}{l}\text { Gestational week at diagnosis of } \\
\text { DM }\end{array}$ & $24.97 \pm 7.5$ & $24.39 \pm 8.73$ & 0.3933 \\
\hline Vaginal Delivery & $43.75 \%, N=7$ & $58.06 \%, N=54$ & 0.2891 \\
\hline Cesarian Delivery & $56.25 \%, N=9$ & $41.93 \%, N=39$ & 0.2887 \\
\hline Male offspring & $68.75 \%, N=11$ & $48.39 \%, N=45$ & 0.1341 \\
\hline Female offspring & $31.25 \%, N=5$ & $51.61 \%, N=48$ & 0.1341 \\
\hline Maturity testing upon delivery (week) & $37.59 \pm 2.64$ & $38.5 \pm 1.03$ & 0.1063 \\
\hline Neonatal birth weight $(\mathrm{Kg})$ & $2.78 \pm 0.7$ & $2.98 \pm 0.45$ & 0.1431 \\
\hline Small for Gestational Age & 0 & $5.38 \%$ & 0.3444 \\
\hline Large for Gestational Age & 0 & 3.23 & 0.4681 \\
\hline
\end{tabular}

p-value $<0.05$, significant

for gestational age (LGA), disproportionately LGA, prematurity, lower cord blood glucose and male gender. Mother-related significant risk factors include maternal hyperglycemia, diabetes diagnosed prior to 28 weeks of gestation, pre-pregnancy pregnancy body mass index (BMI) $\geq 25 \mathrm{~kg} / \mathrm{m}^{2}$, maternal diabetes type and glycosylated hemoglobin [1].

In this study, the amount and rate of maternal intravenous glucose administration did not correlate with the development of neonatal hypoglycemia. Likewise, other maternal and infant-related risk factors previously established were not evident in this study. Despite that, there is a significant difference of maternal capillary blood glucose within 6 hours prior to delivery between those whose offsprings developed neonatal hypoglycemia versus those without hypoglycemia, the explanation of which is challenging to elucidate being limited by the retrospective nature of this study.

Moreover, in this study, the average glucose infusion was at $6 \mathrm{~g} / \mathrm{hr}$ with median glucose infused at 18g. In comparison to a previous study by Mendiola et al. [15], it required a glucose infusion of $20 \mathrm{~g} /$ $\mathrm{hr}$ and median amount of $32.5 \mathrm{~g}$ glucose infused to effect neonatal hypoglycemia.

Hyperinsulinemia, being the cause of hypoglycemia, was demonstrated in a study by Milner and Hales [17], wherein umbilical cord plasma insulin levels increased with administration of glucose before delivery. Furthermore, there was a significant correlation with maternal blood glucose and fetal plasma insulin concentration which reached a mean concentration three times that of control. Although this was not evaluated in our study due to the unavailability of data on fetal insulin levels, this can be included in future studies for further evaluation.

In conclusion, based on this study, routine administration of glucose-containing IVF did not influence the incidence of hypoglycemia in neonates born from diabetic mothers. It is recommended however that further prospective studies be conducted to ascertain this relationship. 


\section{Conflict of Interest}

There is no potential conflict of interest of authors that could have inappropriately influenced research and publication of the manuscript.

\section{Acknowledgment}

We thank the UST Section of Endocrinology for all the support they have given and the Medical Records Section of the UST Hospital for accommodating.

\section{REFERENCES}

1. Alemu B, Baydoun $\mathrm{H}$, Olayinka O, et al. Neonatal hypoglycemia in diabetic mothers: A systematic review. Current Pediatric Research. 2017;21(1):42-53.

2. Behrman RE, Kliegman R, Jenson HB. Nelson Textbook of Pediatrics. Philadelphia:W.B. Saunders Co.; 2000.

3. Dawood F, Dowswell T, Quenby S. Intravenous fluids for reducing the duration of labour in low risk nulliparous women. Cochrane Database of Systematic Reviews 2013, Issue 6. Art. No.: CD007715. DOI: 10.1002/14651858. CD007715.pub2

4. Kalra P, Anakal M. Peripartum management of diabetes. Indian Journal of Endocrinology and Metabolism. 2013;17(7):72.

5. Metzger B, Persson B, Lowe L, et al. Hyperglycemia and Adverse Pregnancy Outcome Study: Neonatal Glycemia. Pediatrics. 2010 Dec;126:6.

6. Knip M, Lautala P, Leppäluoto J, et al. Relation of enteroinsular hormones at birth to macrosomia and neonatal hypoglycemia in infants of diabetic mothers. Journal of Pediatrics. 1983 Oct; 103(4):603-11.

7. Grylack L, Chu S, Scanlon J. Use of intravenous fluids before cesarian section: effects on perinatal glucose, insulin, and sodium homeostasis. Obstetrics \& Gynecology. 1984;63(5):654-8.

8. Carmen S. Neonatal hypoglycemia in response to maternal glucose infusion before delivery. Journal of Obstetric, Gynecologic \& Neonatal Nursing. 1986;15(4):319-23.

9. Rosenberg VA, Eglinton GS, Rauch ER, et al. Intrapartum maternal glycemic control in women with insulin requiring diabetes: A randomized clinical trial of rotating fluids versus insulin drip. American Journal of Obstetric and Gynecology. 2006; 195: 1095-9.

10. Diabetes in pregnancy: management from preconception to the postnatal period [Internet]. [cited 2019 Apr 3].
Available from: https://www.nice.org.uk/guidance/ng3/ resources/diabetes-in-pregnancy-management-from-preconception-to-the-postnatal-period-51038446021

11. lafusco D, Stoppoloni F, Salvia G, et al. Use of real time continuous glucose monitoring and intravenous insulin in type 1 diabetic mothers to prevent respiratory distress and hypoglycaemia in infants. BMC Pregnancy \& Childbirth. 2008;8:23.

12. American Diabetes Association Standards of Medical Care in Diabetes - 2017. Diabetes Care. 2017;40(1):S1-S135.

13. Sirimarco M, Guerra H, Lisboa E, Vernini J, et. al. Diagnostic protocol for gestational diabetes mellitus (GDM) (IADPSG/ADA, 2011): influence on the occurrence of GDM and mild gestational hyperglycemia $(M G H)$ and on the perinatal outcomes. Diabetology \& Metabolic Syndrome [Internet]. 2017. Available from: https://dmsjournal.biomedcentral.com/track/pdf/10.1 186/s13098-016-02002 ? site=dmsjournal.biomedcentral.com

14. Mayaan-Metzger A, Lubin D, Kuint J. Hypoglycemia rate in the first days of life among term infants born to diabetic mothers. Neonatology. 2009;96:80-85.

15. Mendiola J, Grylack L, Scanlon JW. Effects of intrapartum maternal glucose infusion on the normal fetus and newborn. Anesthesia \& Analgesia. 1982;61:32-5.

16. Iwasaku K, Tosaki M, Higashi Y, et al. Effect of glucose-containing intravenous fluid administered before cesarian section on maternal and neonatal blood glucose levels. Advances in Obstetrics and Gynecology. 1992;44(4):351-6.

17. Milner R, Hales C. Effect of intravenous glucose on concentration of insulin in maternal and umbilical-cord plasma. British Medical Journal. 1965:1:284-6.

(i) Open Access This article is licensed under a Creative Commons Attribution 4.0 International License, which permits use, sharing, adaptation, distribution and reproduction in any medium or format, as long as you give appropriate credit to the original author(s) and the source, provide a link to the Creative Commons license, and indicate if changes were made. The images or other third party material in this article are included in the article's Creative Commons license, unless indicated otherwise in a credit line to the material. If material is not included in the article's Creative Commons license and your intended use is not permitted by statutory regulation or exceeds the permitted use, you will need to obtain permission directly from the copyright holder. To view a copy of this license, visit http://creativecommons.org/licenses/ by/4.0/. 